\title{
Kinematical Analysis on the Forehand Stroke Technique of Elite Tennis Player Aarno Ruusuvuori
}

\author{
Li Yanliu Zhou Jihe
}

Chengdu Sport University, Chengdu, China,610000

Keywords: Aarno Ruusuvuori ; tennis; forehand stroke; kinematics

Abstract: Forehand stroke technique is the most frequently used and aggressive technique in tennis competition. It's also the first technique for beginners to learn. With the correct, standard and powerful forehand technique, beginners can not only occupy the active position, but also can lay a good foundation in the learning of other technical actions. This paper analyzes the forehand stroke of Finland junior tennis player Aarno Ruusuvuori .The purpose of the paper is to explore the kinematic characteristics and regularities of forehand striking skills of excellent young tennis players, and provide some theoretical references for the tennis lovers, provide reference to learn forehand stroke theory.

\section{Study Object}

The object of this study is the junior Finland tennis athlete Aarno Ruusuvuori, which Won the championship of ITF Junior Master in 2017, basic information of the athlete is shown in Table 1.

Table 1 Basic information of Aarno Ruusuvuori

\begin{tabular}{cccc}
\hline Name & Nationality & Dominate hand & Ranking \\
\hline Aarno Ruusuvuori & Finland & Right & Champion of ITF Junior Master 2017 \\
\hline
\end{tabular}

\section{Study Method}

The main method of the paper is three-dimensional video analysis:Two JVC-GC-PX10AC highspeed cameras (50fps) were used in the ITF Junior Master in 2017 to shoot Aarno Ruusuvuori 's technique movements. The axis of the two cameras is about $50^{\circ}$. The selected video was the last forehand stroke in the 2nd point of the first games first set. Inputting the selected video part in the 3-D Signal TEC V2.0C analysis system. Choosing the Dempster manikin (with 16 links, 21 joint parameters). In the meantime, adding the racket session for the study. Using the step by step analysis method and the low-pass filter to smoothing process to point data and get the result.

\section{Study results}

\section{The definition of the moments, division of stages}

Definition of the moments

T1 The moment of the end of preparation: The moment that feet are split step and backswing.

T2 The moment of backswing: The moment that the racket hand behinds the farthest.

T3 The moment of touching the ball: The moment that the racket is going to touch the ball.

T4 The moment of end of follow through: The moment that the angle is the maximum between shoulder and hip.

Division of stages

T1-T2: Backswing stage,

T2-T3: Swing stage,

T3-T4: Follow through stage.

3.1 Analysis of the Backswing stage

The backswing stage means the process that the player is prepared and backswing. Under the non- 
forced circumstance, the player would have more time to adjust the body position, and to determine the hit line to improve the quality of return. Before adjusting the position, normally player have the split step movement to adjust the dance and backswing.

When Aarno Ruusuvuori in a passive state, he need to move back to ensure a suitable ball position and a faster shooting speed to ensure the accuracy of batting, due to the opponent deep ball back, Aarno Ruusuvuori needs to retreat two steps to adjust the station hit the ball in order to better meet the ball. At the moment of the end of preparation, the left hip angle was $149.2^{\circ}$, the right hip angle was $136.2^{\circ}$, the left knee angle was $134.0^{\circ}$ and the right knee angle was $111.8^{\circ}$. The left ankle angle was $135.0^{\circ}$, the right ankle angle of $97.6^{\circ}$, indicating that at this time, Aarno Ruusuvuori had a lower height of center of gravity, thus provide a strong elastic potential for the next stage. At the moment that the racket hand behinds the farthest, the right shoulder and right elbow angles were $60.7^{\circ}$ and $93.3^{\circ}$, respectively, and the right shoulder angle and right elbow angle were $91.0^{\circ}$ and $119.7^{\circ}$ respectively. The substantial changes of right shoulder drive elbows and wrists so that the racket could reach a reasonable shooting condition,showing the principle that large joints drive small joints. The speed of right shoulder, right elbow, right wrist and head were $1.89 \mathrm{~m} / \mathrm{s}, 2.62 \mathrm{~m} / \mathrm{s}$, $3.71 \mathrm{~m} / \mathrm{s}$ and $8.33 \mathrm{~m} / \mathrm{s}$, respectively, from preparation to shooting to ending at $0.88 \mathrm{~s}$.The right arm moves fast during the shooting, providing a relatively sufficient time for timely forward batting.

\section{Analysis of the Swing stage}

The swing stage refers to the process from the backswing to the moment to contact the ball. This is the functional action in the forehand stroke, and it is the core action of the forehand technique. In this stage, the players need to push off ground to get a larger angular velocity quickly and adequately, driving the hitting-arm to finish the stroke.The shoulder-hip angle of the athlete at the moment that the racket hand behinds the farthest was $-38.4^{\circ}$, the shoulder angle was $9.4^{\circ}$ and the torso rotation was $47.8^{\circ}$. The duration of the phase is $0.16 \mathrm{~s}$. Right knee at the end of this moment is $111.8^{\circ}$,while in the moment that the racket is going to touch the ball,the right knee angle is $162.2^{\circ}$, by the bending to stretching, and the right foot pedal force together to promote the formation of angular kinetic energy. During the batting stage, the angle of shoulder and hip rotation is larger, and the time used is shorter. It indicates that the athlete's right foot is strong enough. The rotation of the body is the energy gained by pedaling the ground, and then the waist can drive the shoulder to get the acceleration of the racket's forward hitting.Repeatedly watching the video, we can see that Aarno Ruusuvuori hit the ball late in the process of hitting, hit the ball by the point of impact on the quality of shots. The right wrist angles at $0.02 \mathrm{~s}, 0.04 \mathrm{~s}$ and $0.06 \mathrm{~s}$ at the racquet ball touch and at the touch were $129.7^{\circ}, 145.6^{\circ}, 161.6^{\circ}$ and $172.4^{\circ}$, respectively. Wrist angle gradually wider, indicating that the wrist is doing active force and forward the ball, from the moment that the racket touches the ball to $0.06 \mathrm{~s}$, the right wrist angle changes is $42.7^{\circ}$, indicating that the player turns his wrist over quickly and whirl the wrist, which helps to keep a fast, steady top spin of the ball in a late shot. When the racket touches the ball, the right shoulder, right elbow, right wrist and head speed were $1.89 \mathrm{~m} / \mathrm{s}, 4.33 \mathrm{~m} / \mathrm{s}, 8.00 \mathrm{~m} / \mathrm{s}$ and $23.14 \mathrm{~m} / \mathrm{s}$, respectively. At the touchdown time, the head speed reached the maximum speed $(23.14 \mathrm{~m} / \mathrm{s})$, indicating at the swing stage, that player swing faster, and action is more stretcher, smoother, nonstop, resulting in a faster head Speed, and improving the hitting effect. [1]At this stage, the time of drawing in place, the angles of the left shoulder, left elbow and left wrist are $99.6^{\circ}, 131.4^{\circ}$ and $147.3^{\circ}$ at the end of shooting, and the angles of the left shoulder, left elbow and left wrist are $81.3^{\circ}, 36.1^{\circ}$ and $123.7^{\circ}$, the rotation angle of the left shoulder, the left elbow and the left wrist is 18.3, 95.3 and 23.6 degrees, and the rotational amplitude is large, which provides the basis for the steady stroke - the balance state.

\section{Analysis of the Follow-through stage}

"Follow-through" means follow the previous action, make sure you finish all the movements and take the racket through the body to the sideways. The follow-through stage refers to the process from the moment of contact the ball to the end of the follow-through action. This action is also one 
of the essential stage in the forehand. The associated kinematic parameters of this stage are signs of the momentum transmission when swing the racket to contact the ball. Aarno Ruusuvuori rightshoulder angle of the racket at the moment of contact with the racket and the end of the swing time were $38.7^{\circ}$ and $111.7^{\circ}$, respectively, and the right-hand elbow angles were $118.1^{\circ}$ and $68.3^{\circ}$, respectively. At this stage, with the increase of the right shoulder angle, the right elbow angle becomes smaller, indicating that during the stage with the turnaround Aarno Ruusuvuori turn shoulder, turn the right elbow so that the racket wrapped ball better, turn swivel shoulder to increased Racket effect on the ball strength and time.Aarno Ruusuvuori time to play with the swing time $0.30 \mathrm{~s}$, (excellent athletes with $0.38 \mathrm{~s}$ time) is shorter, is due to the forward batting stage, the forced opponent can't push the ball forward and increase the kinetic energy at the stage of follow up. Instead, he chooses to take the shot to ensure the stability and rotation of the batter. In a relatively passive state, it is not easy to play the top spin with a strong spin.

\section{Study Conclusions and Suggestions}

\section{Conclusions}

Backswing stage:Aarno Ruusuvuori forced the opponent to the ball under the action faster (stage takes $0.88 \mathrm{~s}$ ), provides a relatively ample time for the next stage.

Swing stage: On the forehand stage, Luther Wali made full use of trunk strength (trunk rotation range of $47.8 \mathrm{DEG}$ ), swing speed (racquet head speed of $23.14 \mathrm{~m} / \mathrm{s}$ ), and quickly turn the wrist, provides a good balance for a stable shot, hit fast and stable topspin.

4.1.3 Follow through stage: Due to the swing stage, the station is not reasonable enough, the swing time of Aarno Ruusuvuori was 0.30s (with excellent athletes $0.38 \mathrm{~s}$ time), which is shorter than the excellent athletes, which indicate that he is not fully push forward to increase kinetic energy.

\section{References:}

[1] LI Zu-qing, ZHOU Ji-he.A three-dimensional kinematic analysis of Simona Halepi ${ }^{-} \mathrm{s}$ forehand rotation technique [J]. Chengdu Sport University, 2016 (7): 155-157

[2]National Institute of Physical Education Textbook Committee. Sports Biomechanics [M] Beijing: People's Sports Press .2005.6

[3] Yang Tao, Zhou Jihe. Andy Murray tennis forehand rotation technical analysis of kinematics [J]. Chengdu Sport University, 2015 (12): 247-249

[4]Ma Yan-hui. Biomechanical Analysis of Touching Action in Tennis Forehand [J]. Yangtze Normal University, 2011 (3): 24-26

[5]SUN Yan-ming, CHANG Chun-fang.Kinematics analysis of the technical characteristics of tennis forehand spinball [J]. Journal of Capital Institute of Physical Education, 2014 (2): 189-192. 